\title{
REDUCIR ROTACIÓN: PROPUESTA DE UN MODELO TEÓRICO PARA MINIMIZAR COSTOS
}

\author{
Sarahi Guillén Ramirez ${ }^{I}$ \\ Héctor Jafet García Pérez ${ }^{I}$ \\ Diana Mireya Nieto Hipólito ${ }^{2}$ \\ ${ }^{I}$ Universidad Autónoma de \\ Ciudad Juárez \\ ${ }^{2}$ Universidad Tecnológica de \\ Ciudad Juárez
}

Recibido: 26 de enero de 2018 Aprobado: 22 de octubre de

2018.
RESUMEN

71 recurso humano es

$\checkmark$ uno de los principales

elementos que otorga

competitividad a las empre-

sas, pues los conocimientos, el liderazgo, la capacidad para la toma de decisiones y la solución de problemas son fundamentales para el logro de metas organizacionales. Por ello, una buena identificación, selección, contratación y permanencia del personal contribuye a una maximización de utilidades. De ahí que la presente investigación muestre una revisión teórica y análisis de los factores involucrados en la rotación del personal, con el propósito de ofrecer un modelo teórico que incida en la reducción de gastos.

Palabras clave: Rotación de personal, satisfacción laboral, costos.

\section{Abstract}

The human resource is one of the main ele-

ments that gives competitiveness to companies, because knowledge, leadership, the ability to make decisions and solve problems are fun- 
damental to the achievement of organizational goals. Therefore, a good identification, selection, recruitment and retention of staff contribute to maximizing profits. Hence the present investigation following a theoretical review and analysis of the factors involved in the rate of turnover, in order to provide a theoretical model that affects the cost minimization.

Keywords: staff turnover, labor satisfaction, costs

\section{INTRODUCCIÓN}

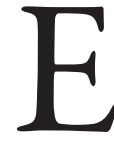

1 recurso humano es uno de los principales elementos que favorecen la competitividad de las empresas, pues son fundamentales los conocimientos, el liderazgo, la habilidad para tomar decisiones y la capacidad para resolver problemas; de ahí la necesidad de identificar al personal adecuado para el logro de metas organizacionales (Zimmerman y Frank, 2006). Desde hace algunos años, las empresas se han enfocado en ser más competitivas y permanecer dentro del mercado, a la par con esto, las organizaciones se han olvidado de la importancia del capital humano y se han enfocado más en la tecnología, sin considerar que dicha situación y los cambios propios del ambiente laboral pueden afectar la relación del empleado y su lugar de empleo (López, 2011).

Con la llegada de la tecnología, las empresas relegaron el capital humano y se enfocaron en conseguir una ventaja competitiva a partir de la reducción de costos. De esta manera, las industrias generaron una fuerte oferta de empleo, ocasionando que las personas no tomen con seriedad su trabajo, o decidan buscar otro empleo con un mejor salario, incrementado así la rotación de personal (RP).

De acuerdo con García (2013), desde 1910 en Estados Unidos se descubrió la necesidad de atender el problema de rotación de personal, debido al costo significativo que representaba contratar personal de manera temporal. Empleadores que solían despedir a sus trabajadores debido a un simple error, y seguros de poder reemplazarlo, se percataron de que cada despido tenía un costo, y de que esa cantidad no se podía pasar por alto. Esto aplicaba igualmente para los empleados que renunciaban a los cargos, de esta manera el número de despidos y las renuncias voluntarias se debían mantener al mínimo posible, para evitar pérdidas en la organización.

Además, González (2006) considera que la rotación de personal es uno de los fenómenos laborales que impactan negativamente la productividad en algunos sectores de la economía, al representar la pérdida de recursos financieros y humanos difícilmente recuperables, lo que implica renovar ciclos de capacitación, curvas prolongadas de aprendizaje y en ese camino, el desperdicio de recursos técnicos e insumos. De igual forma, Buentello y Gómez (2015) concuerdan con el razonamiento anterior, complementando también que, con el tiempo es mayor la dificultad de cubrir los puestos con menor jerarquización y de bajo salario. Un ejemplo de ello es un estudio realizado por ManpowerGroup (2015), el cual muestra los 10 puestos más difíciles de cubrir en México: en primera instancia están los representantes de ventas, des- 
pués las secretarias o asistentes administrativos, trabajadores certificados en algún oficio, ingenieros, operadores de maquinaria/producción, técnicos, gerentes de ventas, gerentes/ejecutivos... y en décimo lugar personal de tecnología de la información.

La baja de empleados (rotación de personal) implica retribuir con mayor remuneración a los nuevos colaboradores para mantenerlos satisfechos dentro de la organización, ello genera un incremento en los costos de inversión del capital humano (Andrade, 2010). Debido a los costos generados por el incremento en la RP, se ha apostado por mantener empleados satisfechos. $\mathrm{Al}$ respecto, Villegas (2012) comenta que "La rotación de personal se da entre empleados insatisfechos y con un deficiente rendimiento en su trabajo y esto los lleva a pensar en buscar un nuevo empleo, evaluar sus alternativas o anunciar su intención de abandonar la empresa".

Por lo anterior, se considera a la rotación (fluctuación) de personal, un resultado de la insatisfacción de los empleados, lo que causa que las empresas tengan elevados egresos en diferentes aspectos, como por ejemplo, los costos de reclutamiento, de selección, de contratación, capacitación y desvinculación, entre otros. Cabe mencionar que en ocasiones el proceso de reclutamiento externo se realiza por medio de volantes, anuncios y periódicos, entre otros, lo que puede provocar que el porcentaje de fluctuación aumente (García, 2013).

Por otro lado, Tamayo (2016) afirma que la satisfacción laboral es una de las principales metas para los departamentos de recursos humanos en las empresas, ya que es el impacto afectivo de los empleados, producido al realizar una evaluación acerca de los aspectos de la organización. De la misma manera influye en las características de cada empleado, pues ocasiona el acercamiento o el abandono del trabajo.

Por lo anterior, se presenta un estudio que describe los orígenes de la rotación de personal y cómo afecta en las actividades de las organizaciones para luego proponer un modelo teórico que favorezca reducir la rotación del personal, al tiempo de minimizar costos. El documento se compone de tres secciones: en la primera se establece el marco de referencia conceptual, teórico y contextual del estudio. Posteriormente, se revisan la teoría de las relaciones humanas, la teoría de las necesidades de Maslow, la teoría de los factores de Herzberg y la teoría de la expectativa. En la tercera parte del documento se incluye una descripción del modelo teórico propuesto y finalmente, la última sección, se expone una serie de reflexiones sobre el modelo teórico propuesto.

\section{Marco Teórico}

hiavenat (2001) con-
sidera la rotación de
personal como una consecuencia de las salidas de trabajadores y la entrada de los mismos para sustituirlos en sus labores. En la tabla 1, se observan distintos conceptos de la rotación de personal, los cuales dan cuenta del problema presentado en las organizaciones, situación que puede solucionarse, al precisar claramente que el intercambio de personal afecta a la organización y su ambiente (Chiavenato, 2007). Del mismo modo, Schulz (2011) define el tér- 
mino de rotación de recursos humanos como la fluctuación de personal entre una organización y su ambiente.

Para Villegas (2012), la rotación de personal está representada por la cantidad de trabajadores que se incorporan y se separan de una institución.

\section{ORIGEN DE LA ROTACIÓN DE PERSONAL}

lores, Abreu y Badii
(2008) consideran este
fenómeno como uno de los primordiales motivos que afecta la estabilidad, el desempeño y la productividad de los trabajadores, debido a esto es importante encontrar el origen de dicho problema; entre los primordiales factores que favorecen a la RP se encuentran la selección incorrecta, la insatisfacción laboral, una baja remuneración y los motivos personales. Por su parte, Hernández, Hernández y Mendieta (2013) indican que la rotación está influenciada por la desmotivación y la insatisfacción laboral; afirman que los motivos que causan la salida de los trabajadores son principalmente laborales, por lo que, de tomarse las

Tabla 1: Conceptos de Rotación

\begin{tabular}{|l|l|l|}
\hline Autor & Concepto & Ideas principales \\
\hline Rodríguez (1998) & $\begin{array}{l}\text { Proceso de cambio en el cual los } \\
\text { empleados pasan de una organiza- } \\
\text { ción a otra. }\end{array}$ & - Proceso de cambio. \\
\hline Galarza (2000) & $\begin{array}{l}\text { Relación entre el número de } \\
\text { empleados que ingresan y los que } \\
\text { dejan de trabajar en la organiza- } \\
\text { ción. }\end{array}$ & $\begin{array}{l}\text { - Empleados que ingre- } \\
\text { san y dejan la organiza- } \\
\text { ción. }\end{array}$ \\
\hline Chiavenato (2001) & $\begin{array}{l}\text { Consecuencia tanto de salidas de } \\
\text { trabajadores como entradas de los } \\
\text { mismos para sustituirlos en sus } \\
\text { labores. }\end{array}$ & $\begin{array}{l}\text { - Entrada y salida de } \\
\text { trabajadores. }\end{array}$ \\
\hline Robbins (2005) & $\begin{array}{l}\text { Es el retiro voluntario e involun- } \\
\text { tario permanente de una organi- } \\
\text { zación. }\end{array}$ & $\begin{array}{l}\text { - Retiro voluntario e } \\
\text { involuntario. }\end{array}$ \\
\hline Chiavenato (2007) & $\begin{array}{l}\text { La rotación es el volumen de per- } \\
\text { sonas que ingresan y que salen de } \\
\text { la organización. }\end{array}$ & $\begin{array}{l}\text { - Personas que ingresan } \\
\text { yue salen de la organi- } \\
\text { zación. }\end{array}$ \\
\hline
\end{tabular}

Fuente: Elaboración propia.

medidas necesarias, se podría reducir dicho concepto.

De acuerdo con Hernández, Espinoza y Aguilar (2014), la salida de los trabajadores está relacionada con la búsqueda de mejores empleos con mayor remuneración, un alto reconocimiento al trabajo realizado y un medio ambiente más seguro y estable. De igual forma, Villegas (2012) indica que las principales causas por las que el personal se retira de una empresa es por mejor sueldo, cambio de localidad, dificultad de adaptación, ma- las relaciones laborales, horario, etcétera. En cambio, Lara (2001) menciona que los cambios de empleo "por una mejor oferta" no se relacionan única y exclusivamente con mejores salarios.

La fluctuación de personal comprende una gran variedad de ventajas, pero también de desventajas, ya que toda rotación requiere de ajustes entre las personas de la organización y su ambiente; pues este se define por el volumen de empleados que ingresan y por el que sale (Schulz, 2011). 


\section{Tipos DE ROTACIÓN DE PERSONAL}

S egún García (2013), la rotación de personal puede clasificarse en externa e interna. La primera define a aquellos que se marchan de la empresa ya sea de forma voluntaria o forzosa, y la segunda ocurre cuando hay transferencias a otras partes de la organización tanto en la misma ciudad o en otra (también se puede presentar cuando hay ascensos o descensos de los empleados). No obstante, Buentello, Valenzuela y Alanís (2015), citando a García (1985), agrupan las salidas de colaboradores en cinco diferentes tipos de bajas:

1. Biológicas o inevitables, esta se presenta en el momento en que ocurre una muerte, jubilación o incapacidad en la empresa.

2. Socialmente necesarias, se refieren a la necesidad de dar de baja a un trabajador por cuestiones de actos ilegales, como son el fraude o robo.

3. Por motivos personales, estas son causadas por situa- ciones o necesidades propias del empleado.

4. Por motivos laborales, son aquellas que son impulsadas por los trabajadores, por ejemplo, motivos de salario, malas condiciones de trabajo, aprovechamiento de las actividades de los empleados por parte de la institución, entre otras.

5. Por decisión de la propia empresa, esto ocurre cuando la decisión de salida es de la organización y no del trabajador por motivos de indisciplina, ruptura de contratos, vencimiento de contrato, y demás. En ocasiones se puede considerar favorable para la organización.

\section{Ventajas $Y$}

\section{DESVENTAJAS DE LA ROTACIÓN DE PERSONAL}

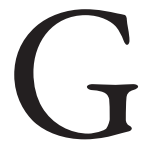

arcía (2013) describe que se pueden encony desventajas en la rotación. Entre las primeras se pueden citar: menos derechos de antigüedad al calcular finiquitos, el nuevo personal puede ser acreedor de un menor salario, algunos puestos requieren de trabajadores jóvenes para una mejor función, entre otras. Al hablar de desventajas se puede hallar: menor eficiencia de los empleados durante su adaptación, el nuevo personal comete más errores, por lo que provoca un alto costo de producción, el proceso de reclutamiento, selección, contratación y entrenamiento simboliza altos costos para la empresa.

\section{SATISFACCIÓN LABORAL}

n la tabla 2 se muestran - distintos conceptos de Lla satisfacción laboral. De acuerdo con las diversas actitudes que se toman, puede ser un factor de agrado o desagrado en relación con el sueldo percibido, su superior y las condiciones de trabajo.

Chiavento (2000) considera que los costos de rotación pueden ser primarios, secundarios y terciarios. Los primarios se relacionan directamente con las actividades de reclutamiento, selección y documentación durante la contratación y desvinculación de los empleados. Los costos secundarios son intangibles, impactan en la productividad y actitudes de los empleados; 
se les considera cualitativos. A su vez, los costos terciarios corresponden a los daños colaterales de la rotación; se reflejan en costos extra de inversión y pérdidas de clientes.

Los costos pueden considerarse en términos monetarios como la inversión realizada por una empresa para adquirir bienes o personal calificado. En la tabla 3 se incluyen los conceptos de costos, según distintos autores. Este es un factor que debe estar bien definido. Es un valor monetario que puede considerarse como inversión cuando se trata de un empleado que posee conocimientos específicos y que resultaría difícil de reemplazar.

\section{Causas Y CONSECUENCIAS DE LA ROTACIÓN DE PERSONAL}

egún Martínez (2010),
hay tres causas de ro-
tación de personal, las
cuales siguen un orden: las de
carácter general, que son inde-
pendientes de la empresa y de
cada persona; de carácter em-
presarial, ligadas a la implanta-
ción de la política del personal;
y las de carácter personal, todas
aquellas relacionadas con el in-

Tabla 2: Conceptos de Satisfacción Laboral.

\begin{tabular}{|l|l|l|}
\hline Autor & Concepto & Ideas principales \\
\hline Locke (1976) & $\begin{array}{l}\text { Un estado emocional positivo o pla- } \\
\text { centero resultante de la valoración } \\
\text { personal que hace el individuo sobre } \\
\text { su trabajo y sobre la experiencia } \\
\text { adquirida en el mismo. }\end{array}$ & $\begin{array}{l}\text {-Estado emocional posi- } \\
\text { tivo o placentero sobre } \\
\text { su trabajo. }\end{array}$ \\
\hline Blum (1990) & $\begin{array}{l}\text { Es el resultado de diversas actitudes } \\
\text { que poseen los empleados. }\end{array}$ & $\begin{array}{l}\text {-Actitudes que poseen } \\
\text { los empleados. }\end{array}$ \\
\hline Robbins (1996) & $\begin{array}{l}\text { Actitud general de un individuo } \\
\text { hacia su trabajo. }\end{array}$ & $\begin{array}{l}\text {-Actitudes positivas } \\
\text { o negativas hacia su } \\
\text { puesto. }\end{array}$ \\
\hline $\begin{array}{l}\text { Davis y News- } \\
\text { trom (2003) }\end{array}$ & $\begin{array}{l}\text { Es el conjunto de sentimientos y } \\
\text { emociones favorables o desfavo- } \\
\text { rables con que los empleados ven } \\
\text { su trabajo. Se trata de una actitud } \\
\text { afectiva, un sentimiento de agrado o } \\
\text { desagrado relativo hacia algo. }\end{array}$ & $\begin{array}{l}\text {-Sentimientos y emo- } \\
\text { ciones favorables o } \\
\text { desfavorables. }\end{array}$ \\
\hline $\begin{array}{l}\text { Hegney, Plank y } \\
\text { Parker (2006) }\end{array}$ & $\begin{array}{l}\text { Es ampliamente determinada por } \\
\text { la interacción entre el personal y las } \\
\text { características del ambiente. }\end{array}$ & $\begin{array}{l}\text {-Interacción entre el } \\
\text { personal y el ambiente. }\end{array}$ \\
\hline
\end{tabular}

Fuente: Elaboración propia.

dividuo, a sus condiciones personales y familiares.

La rotación de personal es un problema que enfrentan las empresas del mundo, por lo que se debe ser meticuloso desde la contratación, el entrenamiento y las responsabilidades que tendrá a cargo el trabajador, así como proveer una capacitación constante para que realice mejor su trabajo, ya que dichos factores representan altos costos para la empresa (González, 2006). Para motivar la disminución de la rotación, los empleados deben sentir que son escuchados por sus superiores, y que sus opiniones son tomadas en cuenta, pues de esta manera pueden expresar sus inconformidades (Aguilar, 2015). 
Tabla 3: Conceptos de costos.

\begin{tabular}{|l|l|l|}
\hline Autor & Concepto & Ideas principales \\
\hline Trettel (1988) & $\begin{array}{l}\text { Remuneración del esfuerzo físico } \\
\text { de los empleados, o inversión para } \\
\text { poseer un personal calificado. }\end{array}$ & $\begin{array}{l}\text {-Inversión y remune- } \\
\text { ración. }\end{array}$ \\
\hline Reveles (2004) & $\begin{array}{l}\text { Información para predeterminar, } \\
\text { registrar, acumular, distribuir, } \\
\text { controlar, analizar, interpretar e } \\
\text { informar costos de producción, } \\
\text { distribución, administración y } \\
\text { financiamiento. }\end{array}$ & $\begin{array}{l}\text {-Sistema de informa- } \\
\text { ción para controlar, } \\
\text { analizar e interpretar } \\
\text { costos. }\end{array}$ \\
\hline García (2008) & $\begin{array}{l}\text { Valor monetario de los recursos a } \\
\text { cambio bienes o servicios que se } \\
\text { adquieren. }\end{array}$ & -Valor monetario. \\
\hline
\end{tabular}

Fuente: Elaboración propia.

\section{LA ROTACIÓN DE PERSONAL EN MÉXICO}

C

1 Estudio Nacional de

Sueldos y Rotación de

Empleo en el Sector de

Tecnologías de la Información, elaborado por la Secretaría de Economía (2016) en México, señala que el nivel de fluctuación del personal depende del tamaño de la empresa. Para las micro y medianas empresas el nivel de rotación se ubica entre el $5.327 \%$ y $5.29 \%$, mientras que las grandes empresas presentan un $0.41 \%$. Cabe mencionar que empresas de una ciudad fronteriza como Tijuana pueden presentar una tasa de rotación anual entre un
29\% y 45\% (González, Orozco y Vizcarra, 2015), las cuales exceden del 15\% recomendable (Arias, 2004). De igual forma, en Ciudad Juárez, en 2015 la rotación anual de personal alcanzó un $5.84 \%$, a diferencia del año anterior donde este índice representó solo un 3.88\%. considerarse que la rotación de personal puede incrementarse debido a una selección incodii y Abreu, 2008).

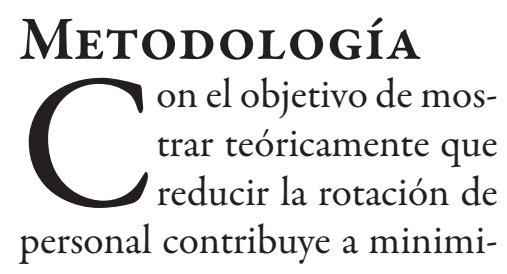
(Gaceta Caseem, 2016). Debe rrecta de personal (Flores, Ba- zar los costos de una organización, se presenta un estudio de tipo documental y descriptivo. La figura 1 muestra el marco referencial del estudio, integrado por un cuerpo teórico, conceptual y contextual de la rotación de personal. Para llevar a cabo la investigación primero se realizó una revisión de los conceptos de rotación de personal, satisfacción laboral y costos. Luego se revisaron las siguientes teorías: de las relaciones, de las necesidades, de los factores y de la expectativa. También se revisaron las causas y consecuencias de la rotación de personal, así como la situación actual de México. Para finalizar, se propuso un modelo teórico que expone la relación entre la satisfacción laboral, la rotación de personal y sus costos.

Entre las teorías con las que se relaciona la rotación de personal, y que fundamentan el modelo propuesto en este estudio, se encuentran las teorías de las relaciones humanas, de las necesidades de Maslow, de los dos factores de Herzberg y de las expectativas, como se aprecia en la figura 2 . 
TeOría de LAS RELACIONES HUMANAS

$n$ Estados Unidos, El-
ton Mayo y sus cola-
boradores John Dewey y Kurt Lewin desarrollaron la teoría de las relaciones humanas, también llamada escuela humanística de la administración. Dicha teoría surgió en 1932 debido a la necesidad de disminuir la tendencia a la deshumanización del trabajo, debido a los métodos rígidos y precisos (Caballero, 2002).

Para Triana (2007), la teoría de Mayo establece seis principios:

1) El primero es la integración y el comportamiento social: elevar la producción depende de la integración social entre los colaboradores, pues un trabajador no actúa por separado.

2) Las recompensas y sanciones sociales: los trabajadores que producen sobre el límite o por debajo de él, pierden el respeto de sus compañeros.

3) Los grupos informales: la confraternidad de los empleados puede sobrepasar el margen formal de la institución, por lo que

Figura 1: Marco de referencia.

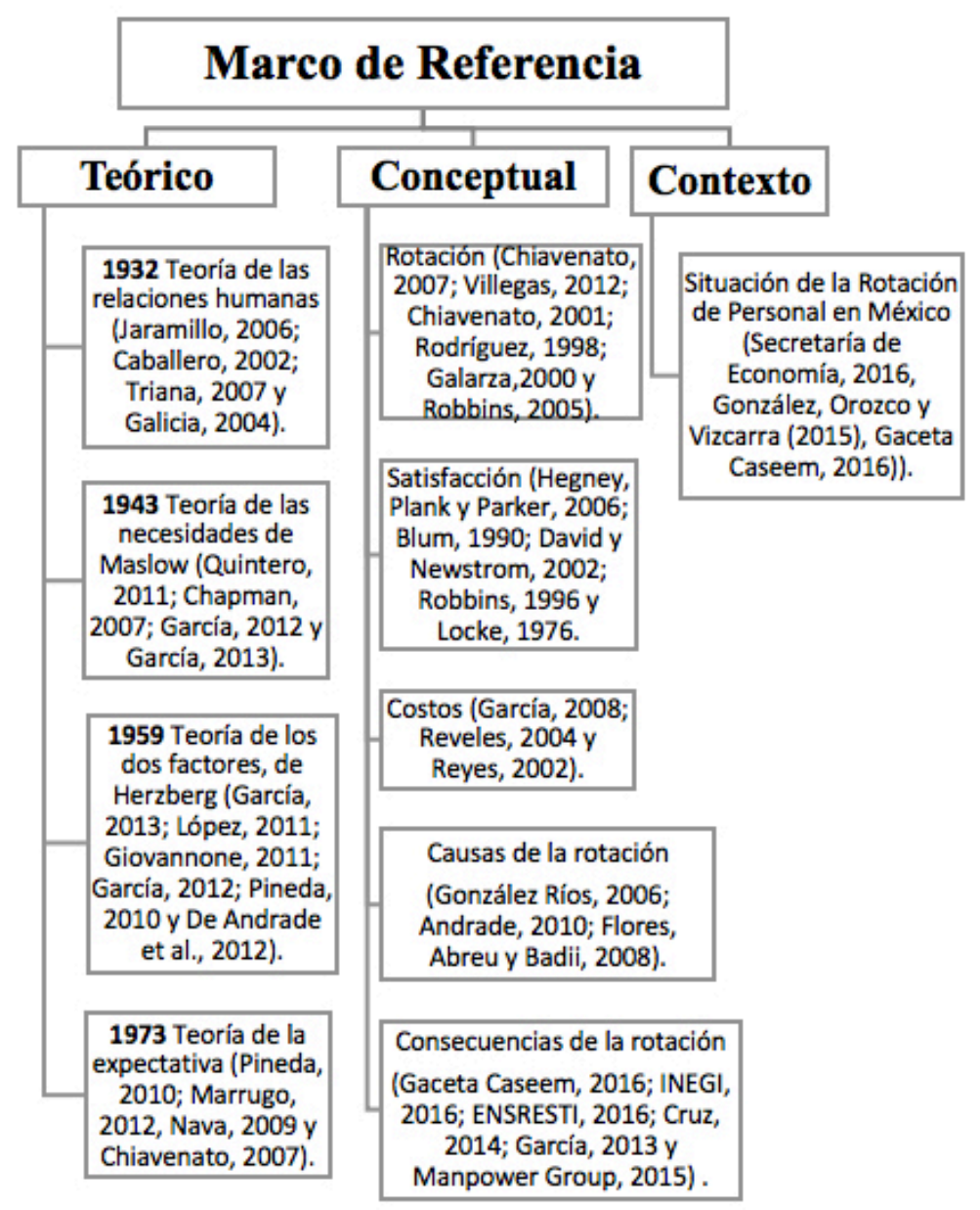

Fuente: Elaboración propia.

en ocasiones ellos mismos definen sus recompensas y sanciones.

4) El contenido del cargo: la preparación específica en un puesto no garantiza la eficiencia.

5) Los aspectos emocionales: las relaciones humanas y la cooperación son puntos importantes para disminuir y evitar conflictos.

6) Estilo de supervisión: los altos puestos deben ser capaces de tratar de manera digna a sus trabajadores, saber comunicar, ser persuasivos y democráticos. 
Figura 2: Teorías que fundamentan el estudio.
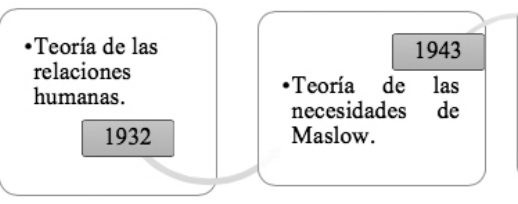
-Teoría de los dos factores de Herzberg.$$
1959
$$

Fuente: Elaboración propia.

\section{TeOría de las NECESIDADES}

$\mathrm{L}$ a también conocida como teoría de la motivación humana, desarrollada por Abraham Maslow en 1943, propone niveles de jerarquización sobre necesidades y factores de motivación para las personas. Esta jerarquía se divide en cinco categorías, considerando su orden de importancia de manera ascendente, de acuerdo con la supervivencia y la capacidad de motivación, como se aprecia en la figura 3 (Quintero, 2011).

Las primeras necesidades son las fisiológicas, todas aquellas que son indispensables para la supervivencia humana. En el segundo escalón se encuentran las de seguridad, que se basan en la protección y los peligros a los que puede estar expuesta una persona. En el siguiente nivel se locali- zan las necesidades de relación social, porque una persona tiende a ser parte de un grupo social, recibir afecto, convivir, comunicarse y sentirse acompañado de otras personas. Después está el ego o estima, que incluye la autovaloración, respeto por sí mismo, no solo el ser integrante de un grupo social. Y por último, las necesidades de autorrealización, cuando las personas intentan desarrollarse tanto en potencial, creatividad y talento, así alcanzan el nivel más alto de sus capacidades personales (García, 2012). La teoría de la motivación humana considera que solo cuando se hayan satisfecho las necesidades de los niveles inferiores de la pirámide, se podrán satisfacer las de los niveles más altos, pues el escalón más alto es la autosuperación (González, 2013).

\section{TeOría de LOS DOS FACTORES n 1959 Frederick Herz-
berg desarrolló la teoría
de los dos factores, en} la que destaca la importancia de las condiciones de trabajo para determinar si es motivante o no. El autor estudió las condiciones de trabajo que representan un medio de motivación (García, 2013). Según López (2011), la teoría se basa en el ambiente externo y en el trabajo del individuo; depende de dos factores:

$>$ Higiénicos, conformados por las condiciones que rodean a la persona al momento de trabajar, desde condiciones físicas como ambientales, el salario, las políticas empresariales, la supervisión, el compañerismo, reglamentos internos, hasta las oportunidades existentes. Tradicionalmente las empresas utilizan la perspectiva ambiental para motivar a los empleados. Los factores higiénicos poseen una capacidad limitada para influir en el comportamiento de los trabajadores; la expresión de higiene refleja el carácter preventivo y muestra cómo 
evitar fuentes de insatisfacción o amenazas que puedan romper el equilibro (Giovannone, 2011). $>$ Motivacionales, que se relacionan con el cargo desempeñado por una persona, como las tareas y los deberes, pues pueden producir un efecto de satisfacción y un incremento de productividad hasta los niveles de excelencia. La motivación incluye los sentimientos, el crecimiento y el reconocimiento profesional, la ejecución de tareas y actividades que sean un gran desafío, ya que son significativos para el trabajo. Los factores motivacionales así como pueden elevar la satisfacción, también pueden provocar una pérdida de la misma (Pineda, 2010).

La teoría de los dos factores afirma que:

1.La satisfacción en el cargo está en función del contenido o de las actividades desafiantes y estimulantes del cargo, considerados factores motivadores.

2.Mientras que la insatisfacción en el cargo está relacionada con el ambiente,

Figura 3: Pirámide de las necesidades.

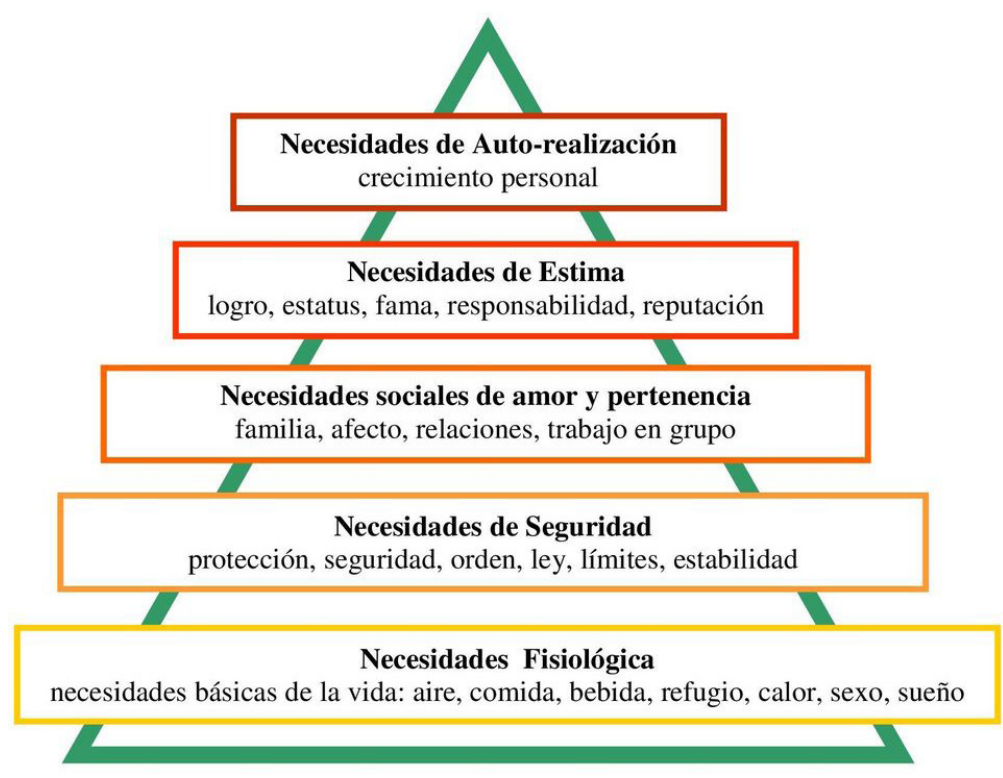

Fuente: Quintero (2011).

la supervisión, los colegas y del contexto en general del cargo, siendo estos factores higiénicos.

Como resultado, Herzberg menciona que el beneficio de las tareas, consiste en aumentar la responsabilidad, objetivos y el desafío de las tareas del cargo (García, 2013).

\section{TEORÍA DE LAS EXPECTATIVAS \\ 7 n 1973, Lawner III du- $\checkmark$ rante el desarrollo de sus trabajos descubrió}

la eficiencia de la remuneración financiera, la cual puede motivar no solo el desempeño, sino también el compañerismo y la dedicación a la empresa, a esto le llamó teoría de las expectativas (figura 5) (Pineda, 2010). Sin embargo, Nava (2009) describe algunas razones que pueden encontrar incoherente esta teoría; entre ellas está la tardanza de recibir un incentivo, ya que crea una falsa impresión de que las ganancias son independientes del desempeño, y esto causa que la relación entre la 
remuneración y el desempeño sea frágil. También se pueden crear falsas expectativas cuando los gerentes no establecen distinciones en las evaluaciones de desempeño, especialmente cuando hay diferencias salariales, pues la confrontación con los trabajadores crea problemas en las relaciones. Y por último, un empleado podría albergar falsas esperanzas debido a las políticas salariales, ya que se basan en criterios gubernamentales, pues los salarios no distinguen el buen o mal desempeño.

Chiavenato (2007) explica que Lawler III concluye su teoría con dos bases:

1. Las personas desean el dinero porque les permite no solo satisfacer sus necesidades básicas y de seguridad, sino también las necesidades sociales, de estima y de autorrealización.

2. Las personas que perciben y creen que su desempeño es necesario para obtener más dinero, se desempeñan de la mejor manera posible.

Esta teoría explica cómo el dinero puede ser un motiva- dor muy poderoso si los trabajadores creen que existe una relación directa o indirecta entre el desempeño y un aumento de remuneración (Marrugo, 2012).

\section{MOdELO TEÓRICO PROPUESTO PARA REDUCIR LA ROTACIÓN DE PERSONAL}

T $\begin{gathered}\text { o expuesto durante la } \\ \text { revisión teórica mues- } \\ \text { tra la relación existente }\end{gathered}$ entre la satisfacción laboral, la rotación del personal y los costos dentro de una organización. Dicha correspondencia puede considerarse positiva. La literatura consultada indica que al reducirse la rotación del personal también se minimizan los costos. Cuando un colaborador se siente satisfecho con las prestaciones económicas recibidas, la relación con su superior y sus condiciones de trabajo, es decir, el bienestar que percibe, puede provocar que evalúe detenidamente las alternativas laborales que se le presentan y no abandone su lugar de trabajo tan fácilmente.
Las teorías revisadas previamente explican cómo influye en el individuo la satisfacción de sus necesidades. Luego de cubrir una o más de ellas, nacen nuevas expectativas, dando como resultado que un individuo busque un ambiente laboral en el que se sienta seguro física, emocional y económicamente, pues de lo contrario, se separará de la empresa, provocando un incremento en los costos y en el índice de rotación.

Por lo anterior, el modelo propuesto contempla tres variables; la satisfacción laboral y de remuneración, índice de rotación y costos generados por la contratación de los empleados. Locke (1976), citado por Pérez y Fidalgo (1999), describe la satisfacción laboral como un "estado emocional positivo o placentero de la percepción subjetiva de las experiencias laborales del sujeto" (p.1). El índice de rotación está representado por el porcentaje de los empleados que fluctúan dentro de una organización y su relación con el promedio de empleados que la conforman (Flores, Abreu y Badii, 2008). Para García (2008) los costos son "el valor monetario 
de los recursos que se entregan o prometen entregar a cambio de bienes o servicios que se adquieren".

La figura 4 muestra el modelo teórico propuesto en este estudio, el cual está enlazado con la rotación de personal, pues la satisfacción laboral es un punto significante para que los trabajadores permanezcan en una empresa; de lo contrario, el índice de rotación aumenta y las empresas son afectadas en los costos. Como se ha visto anteriormente, los egresos de una organización pueden incrementarse debido al índice de rotación de personal, la cual puede aumentar cuando el personal se siente insatisfecho laboralmente. De ahí que el modelo resalte la reducción de costos, cuando los empleados están satisfechos con el sueldo percibido, las relaciones y las condiciones de trabajo.

Con la finalidad de mostrar un reducción en los costos, se han considerado en el modelo aquellas erogaciones fáciles de cuantificar y relacionadas directamente con la rotación de personal, siendo estas las actividades de reclutamiento, selección, contratación, ca-

Figura 4: Modelo teórico propuesto.

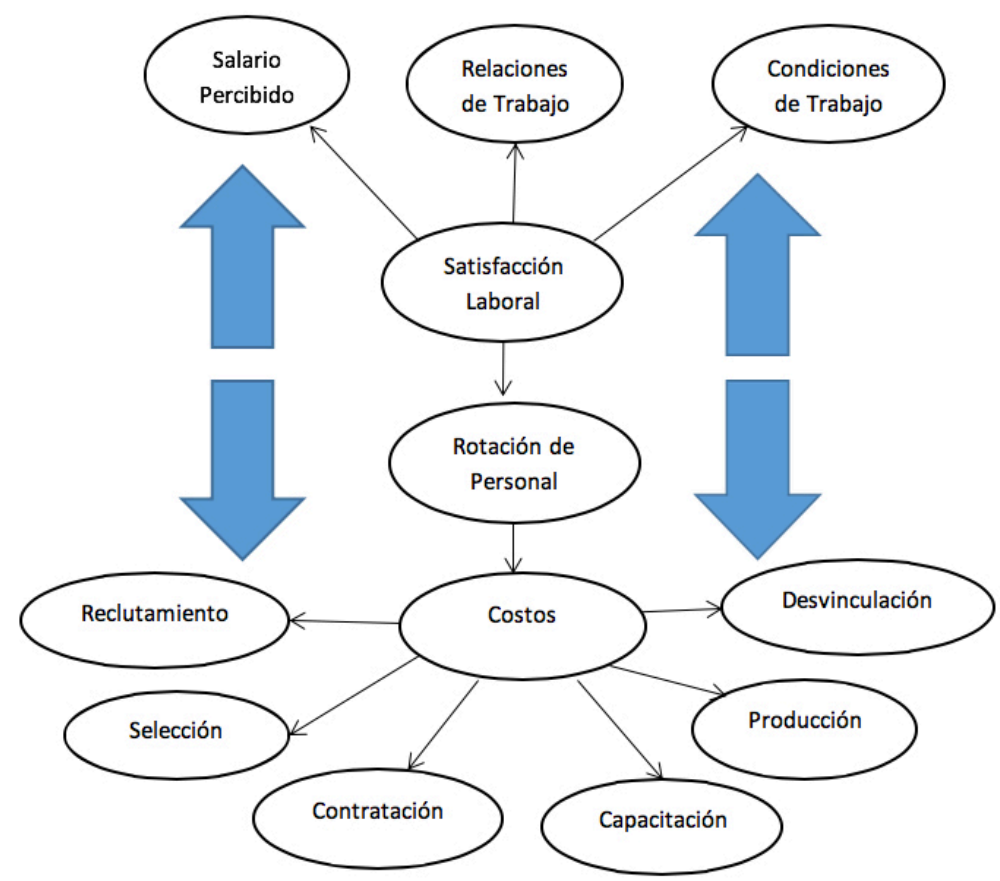

Fuente: Elaboración propia.

pacitación y desvinculación (Chiavenato, 2000).

\section{REFLEXIONES FINALES}

1 modelo propuesto contiene fundamentos teóricos, pero requiere de evidencias empíricas para medir su impacto real en la industria. La literatura consultada muestra que la satisfacción sí influye de manera positiva en el índice de rotación de personal, y este a su vez, en los costos, por lo que se recomienda medir la satisfacción de los empleados de manera sistemática e implementar estrategias para elevar este indicador, comparar con la rotación del personal y los costos.

Cuando un empleado se encuentra satisfecho con el sueldo que percibe, no tomará a la ligera la decisión de cambiar su lugar de trabajo, por lo que resulta conveniente la aplicación de encuestas de salida del personal. Además, un 
sistema de estímulos fomenta en primera instancia la puntualidad y asistencia del personal (Rivero y Almanza, 2001), los cuales a la larga podrían contribuir a reducir los índices de rotación.

Por otro lado, las organizaciones no pueden conformarse con medir los índices de rotación de su personal, sino desarrollar estrategias encaminadas a mejorar las prestaciones, relaciones y condiciones de trabajo. Deben considerar este tipo de acciones como una inversión que a la larga les permitirá reducir sus costos de reclutamiento, selección, contratación, capacitación y desvinculación del personal, lo que a su vez tendrá un impacto positivo en su productividad.

Finalmente, se espera que el modelo teórico propuesto contribuya al diseño de estrategias organizacionales que favorezcan la reducción de costos, contar con empleados satisfechos, así como al logro y mantenimiento de una tasa de rotación recomendable. 


\section{1 novag}

\section{BibLIOGRAFíA}

Aguilar, V. (2015). Propuestas para reducir el indice de rotación en la empresa Saljamx servicios S.A. de C.V.. Tesis inédita. Universidad Nacional Autónoma de México.

Andrade, M. (2010). Análisis de la rotación de personal y elaboración de una propuesta para su optimización en la pasamanería S. A. de la ciudad de Cuenca en el 2009. Universidad Politécnica Salesiana.

Arias, F. (2004). Administración de recursos humanos. México: Trillas.

Blum, M. (1990). Psicologia industrial: sus fundamentos teoricos y sociales (2 ed.). Mexico: Trillas.

Buentello, C., Valenzuela, N. \& Alanis, L. (2015). “Análisis de la rotación de personal en una cadena comercial". Investigación educativa en Latinoamérica, 53-68.

Caballero, K. (2002). El concepto de "satisfacción en el trabajo" y su proyección en la enseñanza . Profesorado, revista de curriculum y formación del profesorado. Vol. 6, no 1.

Chapman, A. (2007). Maslow"s hirarchy of needs Motivational Model, recuperado de: http://www.businessballs.com/maslow.htm, consultado en octubre 2017.

Chávez, Y. H., Chávez, G. H., \& Ramírez, A. M. (2013). Modelo de rotación de personal y prácticas organizacionales. Historia y comunicacion social, 863.

Chiavenato, I. (2000). Administración de Recursos Humanos. Colombia: McGraw-Hill Interamericana S.A. Quinta Edición.

---- (2001). Administración, teoría, proceso y práctica. Tercera edición. Colombia: McGraw-Hill.

---- (2007). Administración de Recursos Humanos, El Capital Humano de las Organizaciones (8 ed.). México: McGraw-Hill.

Davis, K., \& Newstrom, J. (2003). Comportamiento Humano en el trabajo (11 ed.). México: McGraw-Hill.

Flores, R., Abreu, J. L., \& Badii, M. H. (2008). Factores que originan la rotación de personal en las empresas mexicanas. Daena: International Journal of Good Conscience, 3(1), 65-99. 
Gaceta Caseem. (2016). Rotación y ausentismo en la industria maquiladora: datos de AMAC-INDEX. Caseem, 1.

Galarza, J. (2000). Administración Contemporánea. México: McGraw-Hill.

García, J. (2008). Contabilidad de costos. México: McGraw-Hill.

García, C. (2013). Estudio de la relación entre el engagement y la rotación de personal en una cadena de cafeterías ubicadas en la ciudad de Xalapa-Enríquez, Veracruz, México, en el periodo octubre de 2012-marzo de 2013. Universidad Veracruzana.

Garcia, V. (2012). La Motivacion laboral, estudio descriptivo de algunas variables (Tesis de grado). Universidad de Valladolid, Facultad de Ciencias Sociales, Jurídicas y de la Comunicación, Valladolid.

Giovannone, P. (2011). La gestión de la motivación organizacional con el enfoque de la teoria de Herzberg. Un estudio empirico. Universidad Nacional de La Plata. Facultad de Ciencias Económicas.

González, M. (2006). La rotación de personal como elemento laboral que afecta la misión de la organización. Universidad Autónoma de Nuevo León.

González, S., Orozco, I. \& Vizcarra, N. (2015). Rotación de personal en empresas maquiladoras de la zona de Otay en Tijuana BC. VI Congreso Internacional de Contaduría, Administración e Informática Administrativa "Competitividad y Sustentabilidad Empresarial”. FCCA Morelia, Michoacán, 26 y 27 de noviembre de 2015.

Hegney, D., Plank, A., \& Parker, V. (2006). Extrinsic and intrinsic work values: Their impact on job satisfaction in nursing. Journal of Nursing Management, 281.

Hernández, Y., Hernández, G. \& Mendieta, A. (2013). Modelo de rotación de personal y prácticas organizacionales. Historia y Comunicación Social. 18, 837-863.

Hernández, J., Espinoza, J., \& Aguilar, M. (2016). Diferencias en los motivadores y los valores en el trabajo de empleados en empresas maquiladoras. Contaduría y administración, 61(1), 58-83.

INEGI, Instituto Nacional de Estadística y Geografía. (2016). Recuperado en marzo de 2017, de http://www.inegi.org.mx/

Jaramillo. M. (2006). Enfoque humanístico de la administración. Venezuela. http://www. umc.edu.ve/umc/opsu/contenidos/milagros/Unidades/Unidad\%20III/Guia/G.

Locke, A. (1976). The Nature and Cause of Job Satisfaction, in MD Dunnette Handbook of Industrial and Organization. Marvin D. Dunnette.

López, L. (2011). Identificación de los factores que propician la rotación de personal en empresas comerciales ADO región Veracruz. Universidad Veracruzana.

ManpowerGruop. (2015). Estudio ManpowerGroup sobre escasez de talento 2015. Recuperado de: http://www.manpowergroup.es. Consultado en: octubre 2017. 
Martínez, L. (2010). Análisis de la rotación del personal y elaboración de una propuesta para su optimización en la pasamanería S. A. de la ciudad de Cuenca en el 2009. Universidad Politécnica Salesiana.

Marrugo, M., Pérez, B. (2012). Análisis de la Teoría de las Expectativas de Víctor Vroom en el Centro de Emprendimiento y Desarrollo Pedro Romero “Cemprende”. Universidad de Cartagena. Facultad de Ciencias Económicas.

Nava, A. (2009). Elementos a considerar en la motivación de personal en las bibliotecas. Universidad Nacional Autónoma de México. Facultad de Filosofía y Letras. Colegio de Bibliotecología.

Pérez, J., \& Fidalgo, M. (1999). Satisfacción laboral: escala general de satisfacción. Ministerio de Trabajo y Asuntos Sociales de España.

Pineda, M. (2010). Causas de la rotación de personal en una empresa de seguridad privada. Universidad Michoacana de San Nicolás de Hidalgo, 1-25.

Quintero, J. (2011). Teoria de las necesidades de Maslow. Recuperado el 2017, de www.josequintero.net

Ramos, G., \& Triana, M. (2007). Escuelas de las relaciones humanas y su aplicación en una empresa de telecumunicaciones. Scientia Et Technica, XIII(34), 309-314.

Reveles, R. (2004). Costos I. Guadalajara, México: Universidad de Guadalajara, Centro Universitario de Ciencias Económico Administrativas, División de Contaduría, Departamento de Contabilidad.

Reyes, E. (2002). Contabilidad de costos. México: Limusa México.

Rivero, A. \& Almanza, A. (2001). Rotación, estructura de estímulos y aprendizaje tecnológico en la industria maquiladora del norte de México. Región y sociedad.

Robbins, S. (1996). Organizational behavior: concepts, controversies, applications. Prentice Hall.

Robbins, S. (2005). Administración. Pearson Educación.

Rodríguez, A. (1998). Introducción a la psicología del trabajo y de las organizaciones. Madrid: Pirámide.

Secretaría de Economía, (2016). Estudio Nacional de Sueldos y Rotación de empleo en el Sector TI. Reporte final. Recuperado de: https://prosoft.economia.gob.mx/Imagenes/ ImagenesMaster/Estudios\%20Prosoft/AREF_07.pdf. Consultado en octubre 2017.

Schulz, D. (2011). Rotación de personal, el caso Soriana Los Pinos, en el puerto de Veracruz, Veracruz. Universidad Veracruzana.

Tamayo, P. (2016). Percepción y satisfacción laboral como precursores de rotación de personal. Universidad de Granada.

Tomayo y Tomayo, M. (2004). El proceso de la investigación cientifica. Limusa.

Trettel, R. (1998). Costeo y valuación de los recursos humanos. Revista española de financiación y contabilidad, 24. 


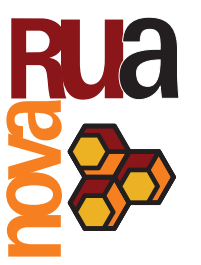

Triana, M. (2007). Escuelas de las relaciones humanas y su aplicación en una empresa de telecumunicaciones. Scientia et technica (34), 309-314.

Villegas, F. (2012). Causas de la rotación de personal de receptores pagadores de la región I metropolitana de una institución financiera. Universidad Rafael Landívar, Facultad de Humanidades, Departamento de Psicología.

Zimmerman, L., \& Frank, H. (2006). Antecedentes de la rotación voluntaria de personal. Red de Revistas Cientificas de América Latina, el Caribe, España y Portugal (97), 7-25. 